\title{
ON THE GENERALIZED HARDY-HILBERT INEQUALITY AND ITS APPLICATIONS
}

\author{
Gao Mingzhe And GaO Xuemei
}

Abstract. A generalized Hardy-Hilbert inequality with weight function of the form $B\left(\frac{p-2+\lambda}{p}\right.$, $\left.\frac{q-2+\lambda}{q}\right)-\theta_{r}(\lambda) /(2 n+1)^{\lambda-\frac{2-\lambda}{r}}$ (with $\theta_{r}(\lambda)>0, r=p, q, 1-\frac{q}{p}<\lambda \leqslant 2, \frac{1}{p}+\frac{1}{q}=1$ and $p \geqslant q>1$ ) can be established by means of Euler-Maclaurin summation formula, where $B(m, n)$ is $\beta$ function. In particular, when $\lambda=1$, an improvement on Hardy-Hilbert's inequality is obtained. As its applications, Hardy-Littlewood's inequality is extended and refined. inequality.

Mathematics subject classification (2000): 26 D15.

Key words and phrases: Hardy-Hilbert's inequality, weight function, $\beta$ function, Hardy-Littlewood's

\section{REFERENCES}

[1] G. H. Hardy, J. E. Littlewood and G. Polya, Inequalities, Cambridge Univ. Press. Cambridge MA, 1952.

[2] YANG Bicheng AND LoKenATH DEBNATH, On a New Generalization of Hardy-Hilbert's Inequality and Its Applications, J. Math. Anal. Appl., Vol. 233, 2 (1999), p. 484-497

[3] Zhang NANyUE, Euler-MacLaurin Summation Formula and Its Applications, Mathematics in Practice and Theory, 1 (1985), p. 30-37. 